\title{
PAEDIATRIC ONCOLOGY IN PAKISTAN: TIME FOR COLLABORATIVE CLINICAL TRIALS
}

Paediatric oncology deals with a diverse group of cancers some of which have cure rates close to $100 \%$ (retinoblastoma and Hodgkin lymphoma) while others are incurable (brainstem gliomas) or have very low survival rates (high-risk neuroblastoma). The past 50 years have seen an unprecedented improvement in survival from childhood cancers. Most cooperative groups are now reporting cure rates exceeding $70 \%$ for paediatric cancers. ${ }^{[1]}$ Better understanding of cancer biology, development of sophisticated response assessment tools and development of newer targeted therapies have contributed to improved survival to a very large extent. In the developed world, treatment-related mortality is becoming rare for most standard treatment modalities. Therefore, cancer-related mortality is confined to relapse and progression of unresponsive cancers. ${ }^{[2]}$

Unfortunately, the success in cure of childhood cancer is not duplicated in resource-poor countries. The reasons are manifold and include factors beyond the control of the paediatric oncologist. Patient-/family-related factors include distance from the treatment facility, malnourishment, poor personal and community hygiene and abandonment of therapy due to many reasons including financial burden. Factors ascribable to poor infrastructure and health system include lack of dedicated paediatric cancer services to ensure early diagnosis and lack of community-based services to support the child on treatment. Even for the minority, who manage to overcome these challenges and are diagnosed and treated in a timely fashion, cure rates are not comparable with those in the Western countries. As expected, cancers requiring only surgical treatment (retinoblastoma and low-grade glioma) and those requiring low-intensity chemotherapy (low stage lymphoma and Wilms' tumour) fair better than those requiring multimodality complex protocols (neuroblastoma, bone and soft tissue sarcoma).

Acute lymphoblastic leukaemia (ALL) is the most common malignancy afflicting children. Improvement in

Correspondence: Dr. Ata Maaz, Dr. Ata Maaz, Department Paediatric Oncology, Noah's Ark Children's Hospital for Wales, Cardigan House, Heath Park, Cardiff, United Kingdom. Email: ata.maaz@wales.nhs.uk the outcome of paediatric ALL has been the prime example of the success of combination chemotherapy. Over the past half-century, ALL has been transformed from a commonly fatal cancer to a highly curable one with the latest studies showing cure rates of above $90 \%$. This success has been a result of a better understanding of disease biology with cancer cytogenetics, development of risk-stratified treatment protocols and sophisticated markers of response; allowing intensification of therapy for poor responders. Most of this success has been a result of a series of carefully conducted clinical trials, each building on the results of the last. ${ }^{[3]}$ Some resource-poor countries have followed this model with encouraging results. ${ }^{[4]}$

Poor outcome of ALL in Pakistan can be ascribed to all of the above factors including patient, health system and treatment-related factors. Enthusiastic adoption of Western protocols without a full comprehension of confounding or prohibitive factors can lead to treatment failure and/or high treatment-related mortality.

Improvement in the outcome of cancer therapy in Pakistani children requires concerted efforts at social and government levels to improve access to cancer care and family support. Prospectively collected outcomes data should become the basis of carefully designed multi-institutional clinical trials. This will help paediatric oncologists build on previous success and lessons learnt will reduce morbidity and mortality during cancer therapy.

Requirements of carrying out reliable research and clinical trials include dedicated clinical trials funding, accurate and timely data collection and adherence to "good clinical practice" that, in turn, necessitates institutional research governance infrastructure. Some of the larger institutions which already have research infrastructure in place can provide leadership in initiating prospective clinical trials.

\section{Ata Maaz}

Department Paediatric Oncology, Noah's Ark Children's Hospital for Wales, Cardiff, United Kingdom Received: 19 March 2015/Accepted: 10 April 2015 


\section{References}

1. The National Registry of Childhood Tumours. Ten-Year Actuarial Survival, Children Aged 0-14 Years, Great Britain, 1971-2005 Data Were Provided by Charles Stiller at the National Registry of Childhood Tumours on Request in 2013. Oxford: NRCT; 2013.

2. Siegel R, Naishadham D, Jemal A. Cancer statistics, 2012.
CA Cancer J Clin 2012;62:10-29.

3. Ching-Hon P, Mullighan CG, Evans WE, et al. Paediatric acute lymphoblastic leukaemia: Where are we going and how do we get there? Blood 2012;120:1165-74.

4. Howard SC, Pedrosa M, Lins M, et al. Establishment of a paediatric oncology program and outcomes of childhood acute lymphoblastic leukaemia in a resource-poor area. JAMA 2004;291:2471-5. 